\title{
Does quicker access to a senior decision maker make a difference in the acute medical unit?
}

\author{
Authors: Hazel Gilbert, Holly Lyne, Shaznin Visanji," Amber Hawksley* and Robert Barker
}

\section{Introduction}

This project aims to reduce the time that clinically stable patients take to be discharged from the acute medical unit (AMU) by improving access to a 'senior decision maker' (defined as ST3 and above). ${ }^{1}$ The patient cohort selected were those referred from their general practitioner (GP) directly to the AMU, of any age, and discharged within 24 hours of attendance, hence avoiding hospital admission. A target of 2 hours to senior review was decided on, as per national guidelines.

\section{Material and methods}

The grade of doctor and time taken to senior review was recorded for 27 patients using the above criteria over 6 weeks. This was compared with the length of time the patients spent in the department before being discharged.

A medical registrar was then moved to the AMU from Monday to Friday from 13.00 to 21.00 to increase access to a senior decision maker and to take pressure off the medical take team based in the emergency department.

\section{Results and discussion}

$59 \%$ of patients had their initial clerking done by a doctor of foundation year 1 (FY1) grade, $24 \%$ by core training years 1 or 2 (CT1/2) and only $7 \%$ by a specialty registrar (SpR).

Time to senior review averaged 3 hours 19 minutes, ranging from 1 hour 32 minutes to 5 hours 45 minutes. Only $17.3 \%$ met the 2-hour target. The time to decision to discharge (DTD) averaged 3 hours 20 minutes, with a range from 16 minutes to 6 hours 52 minutes, meaning $62.5 \%$ met the 4 -hour target.

A dedicated registrar based in AMU improved both the time to senior review and the time to discharge. Time to senior review averaged 2 hours 56 minutes and the time to discharge averaged 2 hours 59 minutes. $47 \%$ met the 2 -hour senior review target and $75 \%$ met the 4 -hour time to discharge target.

\section{Conclusion}

This is one PDSA (Plan, Do, Study, Act) cycle of an ongoing quality improvement project aiming to improve flow of GP-expected patients. These patients have traditionally been seen by the most junior medics and have often had a tortuous journey through the medical department despite the fact that $75 \%$ of them are discharged on the same day.

\section{Reference}

1 NHS Improvement and the Ambulatory Care Network. Same day emergency care: clinical definition, patient selection and metrics. NHS Improvement, 2018:1-15. 